\title{
UM DIAGNÓSTICO DOS CURSOS DE GRADUAÇÃO EM ENGENHARIA DE MINAS E REALIZAÇÃO DE UM COMPARATIVO COM CURSOS DE OUTROS PAÍSES COM GRANDE PRODUÇÃO MINERAL*
}

\author{
Júlia Guimarães Sanches ${ }^{1}$ \\ Maurício Guimarães Bergerman² \\ Carolina Del Roveri ${ }^{3}$
}

\section{Resumo}

O curso de Engenharia de Minas sofreu uma expansão, juntamente com todo o ensino superior no país, por volta de 2005. O objetivo do presente estudo é avaliar as características dos cursos do país e realizar uma comparação com cursos de outros países com grande produção mineral. Foi feito um levantamento inicial a partir de informações disponíveis nos sites do MEC, INEP e das universidades. Como complemento, realizou-se um questionário com os coordenadores dos cursos de Engenharia de Minas do Brasil. Foram compilados ainda dados relativos à estrutura curricular e carga horária dos cursos de Engenharia de Minas dos EUA, Canadá, Austrália, Chile e África do Sul. Em relação à comparação das grades curriculares com os cursos de outros países, observou-se que no Brasil o curso possui uma maior cara horária de Tratamento de Minérios em detrimento da lavra. Nos demais países estudados, o foco das disciplinas é a lavra, já que nestes países a área de Tratamento de Minérios é assunto de outras Engenharias.

Palavras-chave: Educação, Engenharia de Minas.

\section{DIAGNOSIS OF GRADUATION COURSES IN MINING ENGINEERING AND DEVELOPMENT OF A COMPARATIVE STUDY BETWEEN COURSES OF OTHER COUNTRIES WITH GREAT MINERAL PRODUCTION}

\section{Abstract}

The Mining Engineering Undergraduate Course has expanded, along with the higher education as a whole, around 2005. This research aims to evaluate the characteristics of the courses in the country and make a comparison between other countries with high mineral production. A initial survey was made based on information available on the MEC, INEP and universities websites. As a complement, an interview was made with coordinators of Mining Engineering courses in Brazil. Data about curricular structure and the workload of USA, South Africa, Canada, Chile and Australia Mining Engineering courses in USA, Canada, Australia, Chile and South Africa were also analyzed. About the comparison between the Brazilian curricular structure and others' countries, we observe that in Brazil, the course has a longer studyload dedicated to Ore Treatment if compared with Mining Subjects. In other countries analyzed, the focus is on the Mining Subjects, since the Ore Treatment area is usually studied by other Engineering.

Keywords: Education, Mining Engineering

1 Universidade de São Paulo (USP), Departamento de Engenharia de Minas e de Petróleo da Escola Politécnica.email: julia.sanches@usp.br

2 Universidade de São Paulo (USP), Departamento de Engenharia de Minas e de Petróleo da Escola Politécnica. email: mbergerman@usp.br

3 Universidade Federal de Alfenas - Campos avançado de Poços de Caldas. carolina.roveri@unifal-mg.edu.br 


\section{INTRODUÇÃO}

A Engenharia de Minas é um curso de graduação pouco conhecido em todo o Brasil. Até o ano de 2005, havia apenas sete instituições que possuíam o bacharelado em Engenharia de Minas no país (INEP, 2005, 2007). Com a criação do Programa Universidade para Todos (Lei no 11.096, de 13 de janeiro de 2005), aliado ao aumento da produção mineral na primeira década dos anos 2000 , cresceu 0 número de Instituições de Ensino Superior que oferecem o curso. Diversos autores como Sanches, Bergerman e Roveri (2016), Tochtrop Júnior (2015) e Nery e Oliveira (2016) fornecem dados detalhados desta expansão e do aumento do número de vagas ofertadas e formandos em Engenharia de Minas.

Atualmente, a mineração é responsável de três a cinco por cento do PIB no Brasil. Não se observam, no entanto, muitos estudos no setor que avaliem como tem sido feita a expansão dos cursos em Engenharia de Minas e quais as características dos mesmos. Devido a isso, o presente trabalho visa analisar qual o perfil dos cursos do país e fazer um comparativo com universidades do exterior.

\section{MATERIAIS E MÉTODOS}

Inicialmente foram coletados no sistema e-Mec, no site do INEP e em sites sobre universidades do mundo os nomes das instituições de ensino superior que oferecem o curso de Engenharia de Minas no Brasil e nos países que são principais produtores minerais. Contou-se também com o auxílio de alunos e professores que estão nos países ou universidades pesquisadas na busca dos dados internacionais. Em seguida, foram pesquisados nos sites das Universidades do Brasil e do exterior com o curso, suas respectivas cargas e grades horárias e o projeto pedagógico. Em seguida, para obter algumas informações não disponíveis nos sites, foi enviado um questionário eletrônico aos coordenadores dos cursos de Engenharia de Minas do Brasil.

As grades curriculares disponíveis tanto das universidades nacionais quanto das internacionais foram divididas em áreas duas etapas: primeiramente em ensino básico, ensino profissionalizante e ensino específico e posteriormente todas as matérias de ensino específico foram separadas em geologia, lavra e tratamento de minério.

São exemplos das disciplinas classificadas em Geologia: Processos Formadores de Depósitos Minerais, Geoestatística, Mineralogia e Petrologia. Na área de Lavra, considerou-se disciplinas como Mecânica de Rochas, Métodos de Lavra Subterrânea e a Céu Aberto. As disciplinas de Tratamento de Minério incluem Cominuição e Classificação, Flotação e Métodos de Separação. Por fim, os conteúdos que não se encaixam em nenhuma divisão (como Trabalhos de Conclusão de Curso e Estágios Supervisionados) ou em mais de uma das três citadas anteriormente (por exemplo, Economia Mineral, Saúde Ocupacional e Caracterização Tecnológica), foram compiladas como Outros.

\section{RESULTADOS E DISCUSSÃO}

Para o âmbito nacional, os dados base foram retirados do e-Mec e, como são de uma única fonte, foi possível obter a lista completa de cursos oferecidos. Para as instituições internacionais, não foi possível obter dados de todas as Universidades que oferecem o curso. Com os nomes das instituições que contam com o curso no país, pôde-se pesquisar as grades curriculares dos cursos.

As Tabelas 1 e 2 ilustram os resultados obtidos neste levantamento das grades horárias e suas cargas horárias no Brasil. 
Tabela 1. Informações sobre a carga horária.

\begin{tabular}{|l|c|c|c|c|c|}
\hline & Básico & Profissionalizante & Especifico & Outros & Total \\
\hline USP & 1020 & 840 & 2025 & 540 & 4425 \\
\hline UNIFESSPA & 1309 & 1197 & 1275 & 354 & 4135 \\
\hline UFG & 1536 & 432 & 1968 & 228 & 4164 \\
\hline UFPE & 1080 & 705 & 2060 & 180 & 4025 \\
\hline UFBA & 1394 & 986 & 1815 & 272 & 4467 \\
\hline UNIBH & 1160 & 880 & 1811 & 712 & 4563 \\
\hline CEFET-MG & 1082 & 601 & 1792 & 65 & 3540 \\
\hline CETEP & 960 & 390 & 1852 & 30 & 3232 \\
\hline FINOM & 945 & 795 & 1620 & 315 & 3675 \\
\hline UEMG & 1322 & 1000 & 1680 & 240 & 4242 \\
\hline UNIFAL & 2034 & 1124 & 2000 & 36 & 5194 \\
\hline UFOP & 1170 & 720 & 1500 & 160 & 3550 \\
\hline IFES & 1843 & 893 & 1460 & 100 & 4296 \\
\hline UFRGS & 1185 & 945 & 1077 & 200 & 3407 \\
\hline Média & 1289 & 822 & 1710 & 245 & 4065 \\
\hline Porcentagem & 32 & 20 & 42 & 6 & 100 \\
\hline
\end{tabular}

Tabela 2. Divisão do ensino específico

\begin{tabular}{|l|c|c|c|c|c|}
\hline & Geologia & Lavra & Tratamento & Outros & Total \\
\hline USP & 375 & 435 & 345 & 870 & 2025 \\
\hline UNIFESSPA & 104 & 544 & 357 & 270 & 1275 \\
\hline CEULP & 646 & 510 & 272 & 540 & 1968 \\
\hline UFG & 450 & 690 & 220 & 700 & 2060 \\
\hline UFPE & 555 & 420 & 270 & 570 & 1815 \\
\hline UFBA & 612 & 272 & 349 & 578 & 1811 \\
\hline UNIBH & 640 & 440 & 312 & 400 & 1792 \\
\hline CEFET-MG & 425 & 480 & 275 & 672 & 1852 \\
\hline CETEP & 540 & 270 & 300 & 510 & 1620 \\
\hline FINOM & 360 & 240 & 120 & 960 & 1680 \\
\hline FKBH & 440 & 320 & 320 & 920 & 2000 \\
\hline UNIPAC Lafaiete & 340 & 320 & 140 & 700 & 1500 \\
\hline FIP MOC & 535 & 421 & 264 & 240 & 1460 \\
\hline UEMG & 397 & 320 & 177 & 183 & 1077 \\
\hline UNIFAL & 400 & 570 & 180 & 182 & 1332 \\
\hline UFOP & 375 & 240 & 270 & 765 & 1650 \\
\hline FASATC & 480 & 240 & 240 & 630 & 1590 \\
\hline IFES & 520 & 285 & 315 & 529 & 1649 \\
\hline UFRGS & 1110 & 840 & 420 & 470 & 2840 \\
\hline Média & $\mathbf{4 9 0}$ & $\mathbf{4 1 4}$ & $\mathbf{2 7 1}$ & $\mathbf{5 6 3}$ & $\mathbf{1 7 3 7}$ \\
\hline Porcentagem & $\mathbf{2 8}$ & $\mathbf{2 4}$ & $\mathbf{1 6}$ & $\mathbf{3 2}$ & \\
\hline
\end{tabular}

Fonte Tabelas 1 e 2: Elaboração própria, dados dos websites das universidades. 
A maior parte das horas totais dos cursos no geral é da parte específica representando em média $42 \%$ das horas totais. Os dados se alteram ligeiramente quando essas divisões são comparadas entre universidades públicas e privadas. $O$ ensino público tem uma média $400 \mathrm{~h}$ a mais de ensino básico e $300 \mathrm{~h}$ a mais de ensino profissionalizante que as instituições particulares. Isso pode ter interferência sobre a atuação dos profissionais depois de formados. Universidades com um ensino básico mais forte tendem a formar mais pesquisadores que as universidades com uma carga horária mais baixa nessa área.

Nas disciplinas específicas, a diferença da carga horária pode estar ligada ao histórico da universidade, à sua localização e até a presença de outros cursos na mesma instituição como, por exemplo, Geologia e Engenharia Metalúrgica.

Para as universidades internacionais, que estão mostradas na Tabela 3, houve mais dificuldade para encontrar os nomes das instituições que contam com o curso de Engenharia de Minas e na pesquisa das respectivas grades curriculares.

Tabela 3. Divisão do ensino específico das universidades internacionais

\begin{tabular}{|c|c|c|c|c|}
\hline & Geologia & Lavra & Tratamento & Outros \\
\hline \multicolumn{5}{|l|}{ África do Sul } \\
\hline University of Pretoria & 322,5 & 457,5 & 60 & 307,5 \\
\hline \multicolumn{5}{|l|}{ Austrália } \\
\hline University of Adelaide & 600 & 1200 & 120 & 600 \\
\hline University of New South Wales & 480 & 1080 & 0 & 360 \\
\hline University of Queensland & 720 & 1140 & 0 & 240 \\
\hline University of Wollongong & 360 & 840 & 120 & 360 \\
\hline Western Australian School of Mines & 352,5 & 615 & 105 & 120 \\
\hline \multicolumn{5}{|l|}{ EUA } \\
\hline Colorado School of Mines & 210 & 390 & 45 & 285 \\
\hline New Mexico Institute of Mining and Technology & 345 & 300 & 0 & 60 \\
\hline Pennsylvania State University & 270 & 345 & 0 & 180 \\
\hline South Dakota School of Mines and Technology & 195 & 360 & 0 & 330 \\
\hline University of Alaska Fairbanks & 225 & 450 & 0 & 165 \\
\hline University of Arizona & 210 & 465 & 0 & 165 \\
\hline \multicolumn{5}{|l|}{ Canadá } \\
\hline École Polytechnique de Montréal & 195 & 420 & 0 & 375 \\
\hline McGill University & 180 & 630 & 0 & 300 \\
\hline The University of British Columbia & 105 & 420 & 90 & 270 \\
\hline Université Laval & 180 & 420 & 105 & 180 \\
\hline \multicolumn{5}{|l|}{ Chile } \\
\hline Universidad de Atacama & 660 & 1320 & 30 & 570 \\
\hline Universidad de Chile & 450 & 990 & 450 & 990 \\
\hline
\end{tabular}

Fonte: Elaboração própria, dados dos websites

Com as Tabelas 2 e 3 pode-se observar, então, que a área de atuação da Engenharia de Minas se altera de acordo com o país. Na maioria das instituições dos países pesquisados, não há nenhuma matéria de Tratamento de Minérios na grade curricular, ou seja, não entra no leque de atuação da mineração e sim em Engenharia Metalúrgica e Engenharia Química. Devido a isso, a carga horária de Lavra, por exemplo, aumenta de uma média de aproximadamente $401 \mathrm{~h}$ no Brasil 
para 658 h nos outros países para o curso todo. Ou seja, a área de atuação legal do Engenheiro de Minas no país interfere diretamente na carga horária dos cursos.

As principais perguntas do questionário enviado aos coordenadores dos cursos no Brasil estão listadas na Tabela 4.

Tabela 4. Perguntas enviadas às universidades do Brasil

\begin{tabular}{|c|c|c|}
\hline 1 & & $\begin{array}{l}\text { No ano de } 2015 \text { houve a participação de alunos em programas de intercâmbio } \\
\text { (exceto Ciências sem Fronteiras)? }\end{array}$ \\
\hline 2 & & $\begin{array}{l}\text { No ano de } 2015 \text { houve a participação de alunos no Programa Ciências sem } \\
\text { Fronteiras? }\end{array}$ \\
\hline 3 & & $\begin{array}{l}\text { No ano de } 2015 \text { foram concedidas bolsas de Iniciação Científica para os alunos } \\
\text { do curso? }\end{array}$ \\
\hline 4 & & $\begin{array}{l}\text { No ano de } 2015 \text {, houve projetos de extensão vinculados ao curso e/ou aos seus } \\
\text { professores? }\end{array}$ \\
\hline \multirow[t]{3}{*}{5} & & O curso prevê um semestre exclusivo para a realização de estágio? \\
\hline & 5.1 & $\begin{array}{l}\text { Foi observado, nos últimos dois anos, maior dificuldade dos alunos para } \\
\text { conseguir estágios? }\end{array}$ \\
\hline & 5.2 & Há alunos que atrasam sua formação em função da não realização de estágio? \\
\hline 6 & & $\begin{array}{l}\text { Durante o curso, aproximadamente quantos dias de trabalhos de campo ou } \\
\text { visitas são realizados? }\end{array}$ \\
\hline 7 & & $\begin{array}{l}\text { Existe algum programa de acompanhamento dos egressos do curso (para saber } \\
\text { se estão trabalhando na área, por exemplo)? }\end{array}$ \\
\hline \multirow[t]{2}{*}{8} & & O curso possui disciplinas a distância? \\
\hline & 8.1 & $\begin{array}{l}\text { Qual(is) o(s) nome(s) da(s) Disciplina(s) oferecidas a distância e sua(s) carga(s) } \\
\text { horária(s)? }\end{array}$ \\
\hline \multirow[t]{3}{*}{9} & & $\begin{array}{l}\text { Alguma disciplina do curso utiliza a metodologia project based learning (PBL) ou } \\
\text { outra metodologia inovadora? }\end{array}$ \\
\hline & 9.1 & Qual a metodologia empregada? \\
\hline & 9.2 & Qual(is) matéria(s) é(são) lecionada(s) dessa forma? \\
\hline
\end{tabular}

As Tabelas 5 e 6 mostram as universidades que responderam o questionário com suas respectivas informações. As perguntas 5.1, 5.2, 8.1, 9.1 e 9.2 só são feitas às universidades que responderam positivamente as questões anteriores.

Tabela 5. Respostas do questionário

\begin{tabular}{|c|c|c|c|c|c|c|c|c|c|c|c|}
\hline & $\mathbf{1}$ & $\mathbf{2}$ & $\mathbf{3}$ & $\mathbf{4}$ & $\mathbf{5}$ & & & $\mathbf{6}$ & $\mathbf{7}$ & $\mathbf{8}$ & \\
\hline $\begin{array}{c}\text { UNIFAL- } \\
\text { MG }\end{array}$ & $\operatorname{Sim}$ & $\operatorname{Sim}$ & $\operatorname{Sim}$ & $\operatorname{Sim}$ & Não & & & 20 dias & Não & Não & \\
\hline UFOP & $\operatorname{Sim}$ & $\operatorname{Sim}$ & $\operatorname{Sim}$ & $\operatorname{Sim}$ & $\operatorname{Sim}$ & $\operatorname{Sim}$ & $\operatorname{Sim}$ & 30 dias & $\operatorname{Sim}$ & $\operatorname{Sim}$ & $\begin{array}{c}\text { Comunicação e } \\
\text { expressão }\end{array}$ \\
\hline UFPE & Não & $\operatorname{Sim}$ & $\operatorname{Sim}$ & $\operatorname{Sim}$ & $\operatorname{Sim}$ & $\operatorname{Sim}$ & $\operatorname{Sim}$ & 30 a 40 dias & $\operatorname{Sim}$ & Não & \\
\hline UFMT & Não & Não & Não & $\operatorname{Sim}$ & Não & & & 26 & $\operatorname{Sim}$ & Não & \\
\hline $\begin{array}{c}\text { ADJETIVO- } \\
\text { CETEP }\end{array}$ & Não & Não & Não & $\operatorname{Sim}$ & Não & & & 75 dias & $\operatorname{Sim}$ & Não & \\
\hline CEFET-MG & Não & $\operatorname{Sim}$ & $\operatorname{Sim}$ & Não & Não & & & 12 dias & Sim & Não & \\
\hline UFMG & $\operatorname{Sim}$ & $\operatorname{Sim}$ & $\operatorname{Sim}$ & $\operatorname{Sim}$ & Não & & & 20 dias & Não & Não & \\
\hline
\end{tabular}




\begin{tabular}{|c|c|c|c|c|c|c|c|c|c|c|c|}
\hline UFRGS & Sim & Sim & Sim & Sim & Não & & & $\begin{array}{l}12 \text { a } 15 \\
\text { visitas }\end{array}$ & Não & Não & \\
\hline UFBA & Sim & Sim & Sim & Sim & Sim & Sim & Sim & 30 dias & Não & Não & \\
\hline FPAC & Não & Sim & Não & Não & Não & & & 8 dias & Sim & Sim & $\begin{array}{c}\text { Empreendedorismo, } \\
\text { Sociologia, } \\
\text { Filosofia, } \\
\text { Metodologia para o } \\
\text { Trabalho Científico } \\
\text { e Gestão Ambiental. }\end{array}$ \\
\hline UFCG & Não & Sim & Sim & Não & Sim & Sim & Sim & Regularmente & Não & Não & \\
\hline
\end{tabular}

Fonte: Elaboração própria, dados fornecidos pelas universidades.

Tabela 6. Respostas do questionário

\begin{tabular}{|c|c|c|c|}
\hline & 9 & & \\
\hline & & 9.1 & 9.2 \\
\hline $\begin{array}{l}\text { UNIFAL- } \\
\text { MG }\end{array}$ & Sim & $\begin{array}{l}\text { Desenvolvimento de projetos em grupo para } \\
\text { resolução de um problema proposto. }\end{array}$ & $\begin{array}{l}\text { Projeto de Minas I e Projeto } \\
\text { de Minas II }\end{array}$ \\
\hline UFOP & Não & & \\
\hline UFPE & Não & & \\
\hline UFMT & Não & & \\
\hline $\begin{array}{l}\text { ADJETIVO } \\
\text {-CETEP }\end{array}$ & Sim & Project based learning & $\begin{array}{c}\text { Processamento de Minerais } \\
\text { III; Leitura e Produção de } \\
\text { Textos }\end{array}$ \\
\hline CEFET-MG & Não & & \\
\hline UFMG & Não & & \\
\hline UFRGS & Sim & $\begin{array}{l}\text { Laboratório de Realidade Virtual, Laboratórios } \\
\text { computacionais e de tratamento de minérios, } \\
\text { visitas de campo pelos laboratórios de } \\
\text { pesquisa ou disciplinas de graduação, } \\
\text { projetos vinculados a empresas, } \\
\text { oportunizando contato direto do aluno com as } \\
\text { atividades de pesquisa, equipamentos e } \\
\text { metodologias em mineração fora e dentro da } \\
\text { Universidade. } \\
\end{array}$ & $\begin{array}{l}\text { Avaliação de depósitos, } \\
\text { Lavra, Tratamento de } \\
\text { minérios, Geofísica, etc }\end{array}$ \\
\hline UFBA & Não & & \\
\hline FPAC & Não & & \\
\hline UFCG & Não & & \\
\hline
\end{tabular}

Fonte: Elaboração própria, dados fornecidos pelas universidades

Foram enviados questionários para as universidades brasileiras solicitando informações que não estavam presentes nos respectivos sites. Um dado importante foi sobre métodos inovadores de ensino. Com exceção da UFRGS, as faculdades que usam métodos inovadores nas aulas como PBL (Project based learning) ou projetos em grupos, são as com os cursos mais recentes. Ficou facilmente reconhecível que esses métodos inovadores são usados em sua maioria para matérias específicas. Além disso, pôde-se observar que estão sendo implantadas disciplinas à distância, porém de matérias, em geral, profissionalizantes. Sobre as 
visitas técnicas e trabalhos de campo, observou-se um número satisfatório de dias dessas atividades.

\section{CONCLUSÃO}

Os resultados da presente pesquisa indicam uma grande diferença entre as grades curriculares dos cursos de engenharia de minas no Brasil. As universidades públicas contam com maior carga horária no total do curso em relação às particulares, tendo uma diferença maior nas disciplinas básicas do curso. Quando comparadas as grades dos cursos do Brasil com os cursos do exterior, pode-se observar que no país o currículo da Engenharia de Minas é mais amplo, incluindo uma significativa carga horária de Tratamento de Minérios. Nos cursos dos demais países estudados, a formação específica é focada na área de Lavra.

A pesquisa realizada junto aos coordenadores de curso mostrou que as universidades com cursos mais novos são normalmente as que contam com métodos inovadores de ensino, implantados em sua maioria em matérias específicas do curso. Mostrou também que as disciplinas à distância, implantadas em poucos cursos, são de escopo profissionalizante, além do incentivo das universidades em visitas técnicas e trabalho de campo na área.

\section{Agradecimentos}

Agradeço a todas as universidades que responderam ao nosso questionário e nos auxiliaram, assim, na pesquisa: UNIFAL e a senhora Carolina Del Roveri; UFOP e o senhor Carlos Alberto Pereira, UFPE e o senhor Márcio Luiz de S C Barros; UFMT e o senhor Newton Diego Couto do Nascimento; Adjetivo CETEP e o senhor Felipe de Orquiza Milhomen; CEFET Minas e o senhor Hildor José Seer; UFMG e o senhor Roberto Galery; UFRGS e o senhor Paulo Salvadoretti; UFBA e o senhor Erisvaldo Bittencourt de Jesus; FPAC e o senhor Adriano Raimundo Totou; e UFCCG e o senhor José Avelino Freire.

Agradeço também a todas as pessoas que me ajudaram com informações das universidades internacionais como o senhor Aaron Young.

\section{REFERÊNCIAS}

1 DNPM, Departamento Nacional de Produção Mineral [homepage on the internet]. Anuário Mineral 2010. [cited 2016 Mar 27] Available from: http://www.dnpm.gov.br/dnpm/paginas/anuario-mineral/arquivos/ANUARIO_ MINERAL_2010.pdf

2 INEP, Instituto Nacional de Estudos e Pesquisas Educacionais [homepage on the internet]. Sinopses do ensino superior. [cited 2015 Dec 22] Available from: http://portal.inep.gov.br/superior-censosuperior-sinopse

3 MAGALHAES, AOBF. (Org.) ; ROVERI, C. (Org.) ; HORTA, DG. (Org.) ; NAVARRO, FC. (Org.) ; RUSILO, L. C. (Org.) ; BERGERMAN, MG (Org.) ; et al. . Anais do I Workshop de Educação em Engenharia de /minas: rumos da Engenharia de Minas no Brasil. 1. ed. São Paulo: Fishers, 2015. v. 1. 35p . 
4 MARSDEN, J; FRIMPONG, S. The state of mining education; What does the future hold for the next generation. Society for Mining, Metallurgy \& Exploration, organizador. Mining Engeneering Magazine; 2014 Oct vol. 66 no 10 p21-30.

5 MEC, Ministério da Educação [homepage on the internet]. Dados do e-Mec sobre o ensino superior. [cited 2015 Sep 2 and 2015 Sep 8] Available from: http://e-Mec.mec.gov.br/

6 NERY, MAC; OLIVEIRA, MPP; Recursos Minerais no Brasil: Problemas e Desafios. In: Formação de recursos humanos para a mineração. Academia Brasileira de Ciências. Rio de Janeiro, Brasil, 2016. p. 396. [Acesso em 31 mai. 2017]. Disponível em: http://www.abc.org.br/IMG/pdf/doc-7006.pdf

7 ROVERI, C; MAESTRELLI, SC; MARTINS, R; PIRILLO, G; BERGERMAN, MG; NAVARRO, FC ; MAGALHAES, AOBF . Mineração para todos: Programa de divulgação das atividades da engenharia de minas na região de Poços de Caldas, MG. In: XLII Congresso Brasileiro de Educação em Engenharia, 2014, Juiz de Fora. Anais do XLII Congresso Brasileiro de Educação em Engenharia. Brasilia: Abenge, 2014.

8 SANCHES, JG ; BERGERMAN, MG ; ROVERI, C; A Expansão do Ensino Superior no Brasil: Um Diagnóstico dos Cursos de Graduação em Engenharia de Minas. Associação Brasileira de Educação em Engenharia. XLIV Congresso Brasileiro de Educação em Engenharia 2016. Natal, Brasil.

9 TOCHTROP JR, EF; Análise do Crescimento do Número de Cursos de Engenharia de Minas no Período de 2005-2015. XXVI Encontro Nacional de Tratamento de Minério e Metalurgia Extrativa. I Workshop de Educação em Engenharia de Minas. Poços de Caldas, Brasil. 